\title{
Depth-resolved core level spectroscopy of noncentrosymmetric solid BiPd
}

\author{
Arindam Pramanik, ${ }^{1}$ Ram Prakash Pandeya, ${ }^{1}$ Khadiza Ali, ${ }^{1}$ Bhanu Joshi, ${ }^{1}$ Indranil Sarkar, ${ }^{2}$ Paolo Moras $\odot,{ }^{3}$ \\ Polina M. Sheverdyaeva, ${ }^{3}$ Asish K. Kundu ${ }^{0},{ }^{3}$ Carlo Carbone, ${ }^{3}$ A. Thamizhavel, ${ }^{1}$ S. Ramakrishnan, ${ }^{1}$ and Kalobaran Maiti ${ }^{1, *}$ \\ ${ }^{1}$ Department of Condensed Matter Physics and Materials Science, Tata Institute of Fundamental Research, \\ Homi Bhabha Road, Colaba, Mumbai 400005, India \\ ${ }^{2}$ Deutsches Elektronen-Synchrotron DESY, Notkestrasse 85, D-22607 Hamburg, Germany \\ ${ }^{3}$ Istituto di Struttura della Materia, Consiglio Nazionale delle Ricerche, I-34149 Trieste, Italy
}

(Received 7 September 2019; revised manuscript received 14 December 2019; published 29 January 2020)

\begin{abstract}
Understanding exotic solids is a difficult task as interactions are often hidden by the symmetry of the system. Here, we study the electronic properties of a noncentrosymmetric solid, BiPd, which is a rare material exhibiting both superconductivity and the topological phase of matter. Employing high-resolution photoemission spectroscopy with photon energies ranging from the hard x-ray to extreme-ultraviolet regime, we show that hard $\mathrm{x}$-ray spectroscopy alone is not enough to reveal surface-bulk differences in the electronic structure. We derive the escape depths close to the extreme surface sensitivity and find that the photon energies used for high-resolution photoemission measurements fall in the surface sensitive regime. In addition, we discover a deviation of the branching ratio of $\mathrm{Bi}$ core level features derived from conventional quantum theories of the core hole final states. Such a breakdown of the atomic description of the core level spectroscopy can be attributed to the absence of a center of symmetry and spin-orbit interactions.
\end{abstract}

DOI: 10.1103/PhysRevB.101.035426

\section{INTRODUCTION}

Recently, noncentrosymmetric superconductors have attracted a lot of attention for holding the possibility of many unusual phenomena such as mixing of spin-singlet and spintriplet components in superconducting pairs in addition to the topologically protected surface states, which is absent in inversion-symmetric materials. In this class of materials, BiPd has aroused much interest for being a potential candidate for a topological superconductor. BiPd forms in an orthorhombic structure at high temperature with the space group $C m c 2_{1}$, which is called $\beta$-BiPd. Below $483 \mathrm{~K}$, it stabilizes in the monoclinic structure with the space group $P 2_{1}$; it is called $\alpha-\mathrm{BiPd}$, and it does not have inversion symmetry [1]. In this structure, a unit cell consists of two double layers of $\mathrm{Bi}$ and $\mathrm{Pd}$, and both the elements have four nonequivalent sites, as shown in Fig. 1(a). Single crystals of BiPd have a preferential cleaving direction along the $b$ axis. Scanning tunneling microscopy showed that cleaved surfaces are Bi terminated, flat, and free of reconstruction [2]. The small Pd-Pd bond length in this material indicates enhanced $\mathrm{Pd} 4 d-4 d$ hybridization, which might play an important role in deriving the electronic properties of this material. Various bulk measurements established an anisotropic superconducting phase below $3.7 \mathrm{~K}$ [3]. The signature of multiple superconducting gaps has been observed in the point-contact spectroscopy results [4]. Angleresolved photoemission spectroscopy (ARPES) studies have shown a signature of Dirac-like surface states in BiPd [5-7]. Spin-resolved photoemission spectroscopy studies have also

\footnotetext{
*Corresponding author: kbmaiti@tifr.res.in
}

been carried out to confirm the spin polarization of the surface states [5].

While exoticity of the material is evident, the surface character of the electronic states has been explained via the nondispersive nature of the corresponding energy bands as a function of photon energy as out-of-plane crystal momentum $k_{z}$ is directly related to the photon energy. The nondispersive nature of the bands indeed relates to the two-dimensional character of the energy bands typical of the surface states. However, a similar scenario can also occur due to various other localization effects occurring in the bulk such as electron correlation, disorder, spin/charge order, etc. Thus, such a method of characterizing the surface electronic structure may not be unambiguous. Moreover, in a noncentrosymmetric material, the scenario becomes more complex as the absence of a center of symmetry in the bulk crystal structure leads to a potential gradient in the bulk, although it is weaker than that in the surface. Here, we study the electronic structure of BiPd employing high-resolution photoemission spectroscopy while varying the surface sensitivity of the technique; this is a direct method of probing the electronic structure at different depths from the sample surface. Experiments with varied photon energies (ultraviolet to hard $\mathrm{x}$ ray) reveal evidence of a significant difference between the surface and bulk electronic structures in BiPd. In addition, we discover a deviation from the conventional quantum behavior of the core level features, which demands physics beyond the existing paradigm of the core level spectroscopy.

\section{EXPERIMENT}

High-quality single crystals of BiPd were grown using a modified Bridgman method. The crystal structure of the 
sample was determined via analysis of the powder x-ray diffraction pattern, and good crystallinity was ensured by employing Laue diffraction experiments. The lattice parameters found in the study correspond to the monoclinic structure reported elsewhere [1]. Magnetization measurements exhibit a superconducting transition at $3.8 \mathrm{~K} \mathrm{[1].}$

Hard x-ray photoemission measurements (HAXPES) were carried out at the P09 beamline, PETRA III, Hamburg, using a high-resolution Phoibos electron analyzer. The setup was optimized for the best energy resolution of $200 \mathrm{meV}$ found at a photon energy of $5946.6 \mathrm{eV}$. Extreme-ultraviolet photoemission measurements were performed at the VUV photoemission beamline at Elettra, Trieste, using a Gammadata Scienta R4000 WAL analyzer with the energy resolution set to $15 \mathrm{meV}$. The acceptance angle used during the measurements was greater than $30^{\circ}$. Thus, the core level spectra collected in the angle-integrated mode will have little influence due to the photoelectron-diffraction-induced effect. Sample temperature down to $15 \mathrm{~K}$ was controlled using a helium cryostat. The sample was mounted for an easily cleavable $b$ axis after orienting the crystal using the Laue diffraction method and was cleaved in situ to expose the clean surface for experiments. The sample surface was found to be clean, and the reproducibility of the data was verified. The surface sensitivity of the technique was varied in two ways. First, the first is to change the electron emission angle while keeping the photon energy fixed. As the emission angle is increased, photoelectrons from the bulk have to travel a longer path through the sample to reach the sample surface, which diminishes the signal intensity from the bulk, and consequently, the signal from the surface layers gets enhanced. The other method is to vary the photon energy; a change in photon energy results in photoemitted electrons with different kinetic energies and hence different escape depths.

\section{RESULTS AND DISCUSSION}

In Figs. 1(b) and 1(c), we show the $\mathrm{Bi} 4 f$ and $\mathrm{Pd} 4 d$ core level spectra collected at $50 \mathrm{~K}$ in normal emission geometry using HAXPES. There are two sharp and distinct asymmetric peaks in each of the spectra corresponding to the spin-orbit split final states. The asymmetry in the spectral line shape arises due to the low-energy excitations across the Fermi level, which is possible in a system possessing a finite density of states at the Fermi level [8,9]. Thus, the observation of asymmetry reflects metallicity of the material. In addition, there is a broad hump in intensity on the higher-bindingenergy side (shown on an enlarged intensity scale in the inset) in both the spectra. The energy separation of the hump from the main peak is quite similar in both cases, indicating a possible origin related to the energy loss in the final state due to the excitations of the collective oscillation modes.

To establish the above assertion, the spectral features are simulated using a set of peaks representing various photoemission signals; the resulting fit (solid line) is superimposed on the experimental spectra (symbols). It is evident that the humps in the spectra could be captured well by two broad peaks separated by the spin-orbit splitting of the main photoemission signals maintaining their intensity ratio; the fit results are shown in the inset. The energy separation of the loss

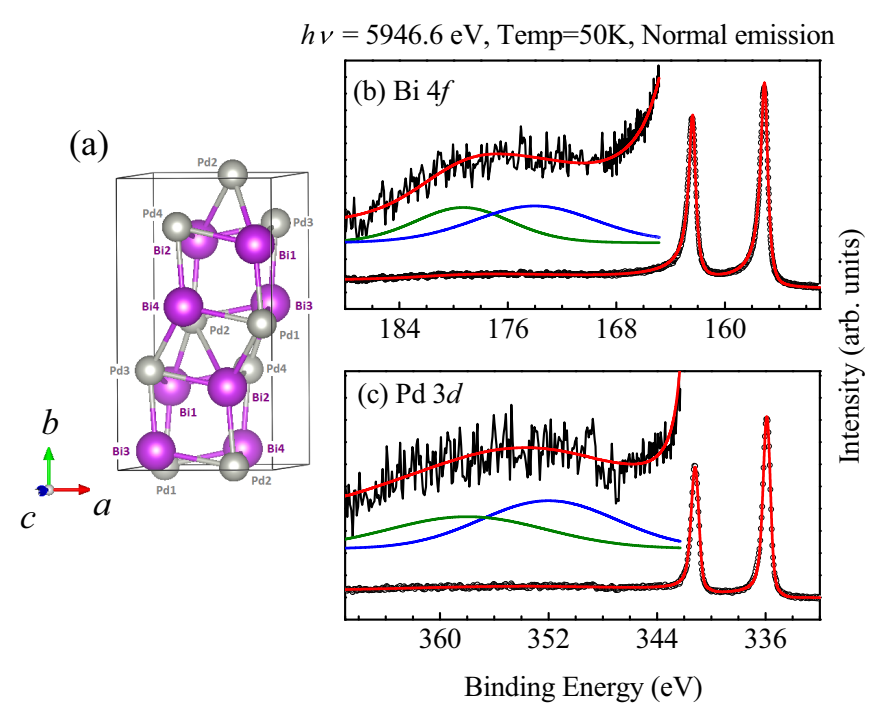

FIG. 1. (a) A unit cell of BiPd (monoclinic structure). Inequivalent $\mathrm{Bi}(\mathrm{Pd})$ atoms are indexed with $1,2,3$, and 4 . (b) $\mathrm{Bi} 4 f$ and (c) Pd $3 d$ HAXPES spectra (open circles). The simulated spectral function is shown by the red line superimposed over the experimental data. Insets show the data (noisy line) in an expanded intensity scale exhibiting a broad hump, which could be simulated by a set of two peaks separated by the spin-orbit coupling of the corresponding core hole.

features from the main peak is of the order of $18 \mathrm{eV}$, which is akin to the bulk plasmon excitation energy found in other systems [10]. Similar loss features are also present in other core level spectra. Since the photoemission process is highly bulk sensitive at 5946.6-eV photon energy, the above features may be considered the bulk property.

The temperature dependence of the bulk spectral functions is shown in Fig. 2; $\mathrm{Bi} 4 f$, $\mathrm{Bi} 5 d$, and $\mathrm{Pd} 3 d$ spectra collected at 50,175 , and $260 \mathrm{~K}$ are superimposed in the figure for better comparison. The peak corresponding to the higher $J$ value is shown in the inset on an expanded energy scale. The Bi $4 f$ and $\mathrm{Bi} 5 d$ spectra are identical at all the temperatures studied. In the case of $\mathrm{Pd} 3 d, 50$ and $175 \mathrm{~K}$ data are identical, but the width at $260 \mathrm{~K}$ is found to be larger by $30 \mathrm{meV}$. Phonon broadening is expected to manifest in all the core level spectra. However, we do not see a broadening-induced effect in $\mathrm{Bi}$ core level spectra, ruling out such a possibility. The other possible effect is the proximity to the structural transition; such a precursor effect associated with the structural transition has been observed in various materials [11]. This can be explained as follows. The width of the core level peak depends on lifetime broadening and screening of the core hole by conduction electrons in the final state. The photoelectron and photohole lifetimes are expected to be less affected by the structural change. Thus, the core level broadening observed here may be attributed to the change in conduction bandwidth as the conduction electrons screening the core hole have a larger degree of itineracy in the orthorhombic phase $(\beta$-BiPd) compared to that in the monoclinic phase $(\alpha-\mathrm{BiPd})$. The effect is most prominent in the $\mathrm{Pd}$ core level as the conduction band is primarily constituted of $\mathrm{Pd} 4 d$ states; the $\mathrm{Bi} 6 p$ contribution is weak. 

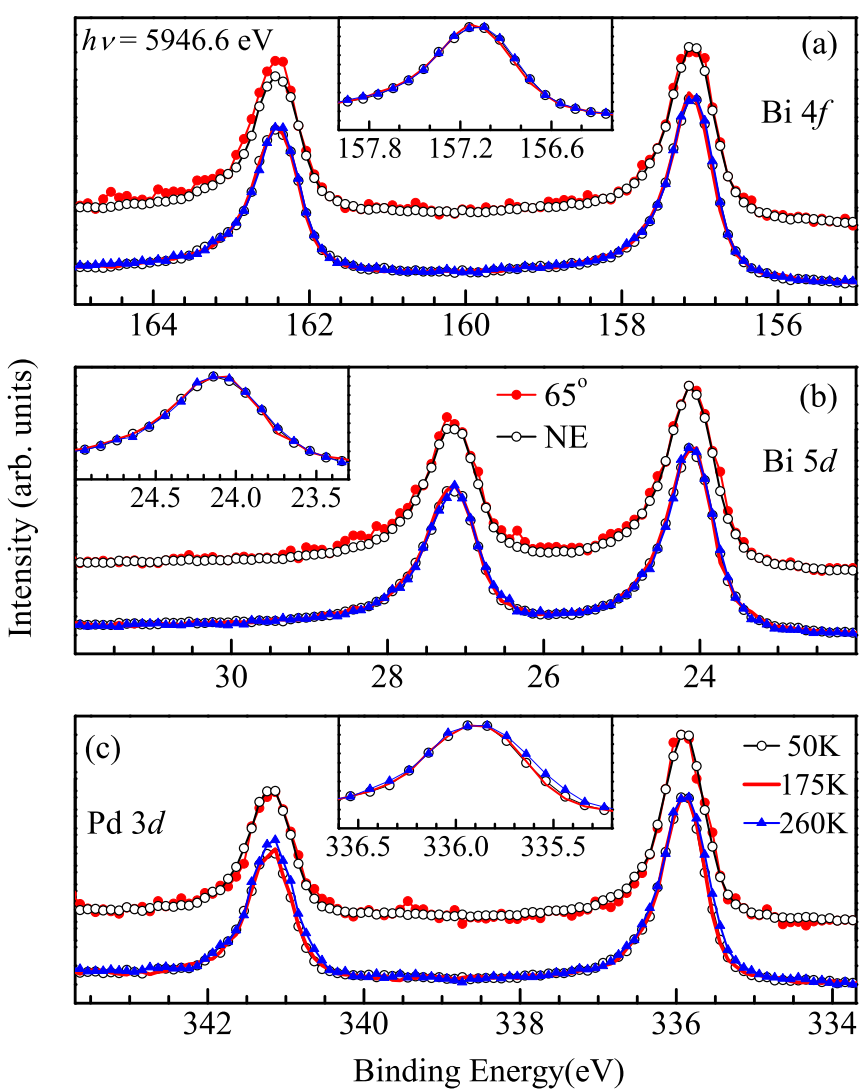

FIG. 2. (a) Bi $4 f$, (b) Bi $5 d$, and (c) Pd $3 d$ core level spectra collected with 5946.6-eV photon energy in normal emission (NE; open circles) and $65^{\circ}$ off-normal emission (solid circles) geometries at a sample temperature of $50 \mathrm{~K}$. Normal emission spectra collected at $50 \mathrm{~K}$ (open circles), $175 \mathrm{~K}$ (line), and $260 \mathrm{~K}$ (triangles) are superimposed. Insets show the high angular momentum peak of the temperature-dependent data in an expanded energy scale.

In order to investigate the surface-bulk differences, we compare the spectral functions collected at normal emission and $65^{\circ}$ angled emission geometries in Fig. 2. The escape depth of photoelectrons with kinetic energy close to $6 \mathrm{keV}$ is about $40 \AA$. At $65^{\circ}$ off-normal emission, the probing depth will become $20 \AA$. In Fig. 2, we show the Bi $4 f$, Bi $5 d$, and $\operatorname{Pd} 3 d$ core level spectra; the data at different emission angles are superimposed. The line shapes of all the spectra are very similar, indicating its insensitivity to this change of probing depth. This suggests that either the surface and bulk electronic structures are similar or the surface electronic structure, if different, must be limited to the top few layers of the sample that could not be probed by this moderate change in surface sensitivity. It is worth mentioning that no distinct signature of inequivalent $\mathrm{Bi}$ and $\mathrm{Pd}$ atoms has been observed in the spectra.

All the core level peaks can be simulated with a DoniachŠunjić line shape using the least-squares error method. The remarkable representation of the asymmetry within the Doniach-Šunjić framework suggests that the asymmetric part originates essentially from the low-energy excitations across the Fermi level. Comparable asymmetries in both Bi and Pd core level spectra provide evidence of finite Bi-Pd hybridization $[12,13]$. Interestingly, while the branching ratio of the $\mathrm{Pd}$ $3 d$ spin-orbit split peaks is in accordance with the multiplicity
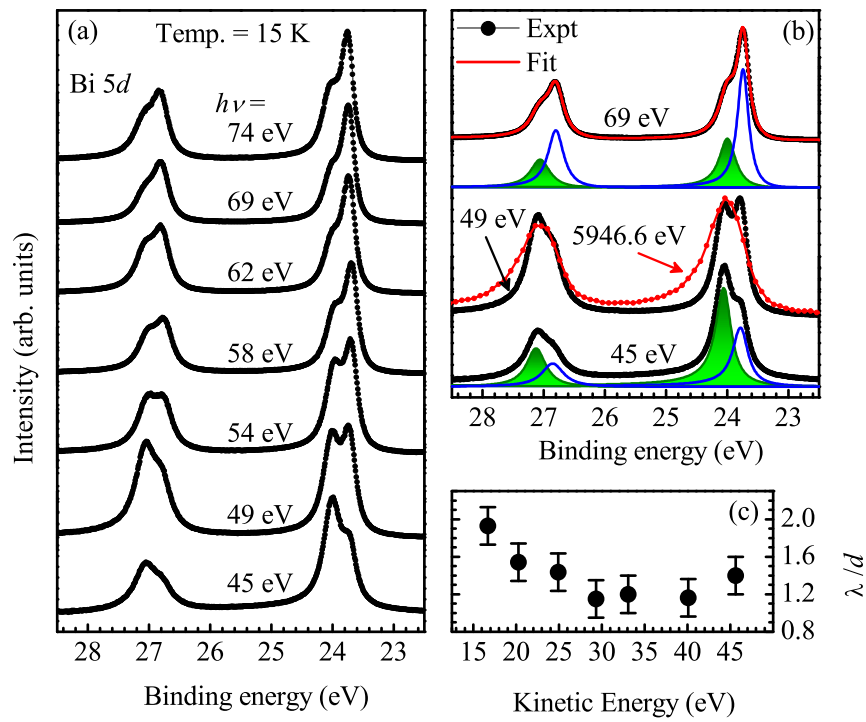

FIG. 3. (a) Bi $5 d$ spectrum collected with different photon energies. (b) Bi $5 d$ spectrum at photon energies of 69,49 , and $45 \mathrm{eV}$. We have superimposed HAXPES data over the $49-\mathrm{eV}$ spectrum. The line and shaded area represent the component peaks constituting the experimental spectra; fitting is done following least-squares error method. (c) The plot of $\lambda / d$ as a function of photoelectron kinetic energy.

of 2:3 of the corresponding final states, Bi core level spectra exhibit a significantly higher branching ratio. The lower limit of the ratio is found to be close to 0.71 for $\mathrm{Bi} 5 d$ and 0.83 for Bi $4 f$, which are significantly higher than the values of 0.67 and 0.75 expected from the multiplicity of the final states-a signature of the deviation from the usual atomic description of the core level spectroscopy. In the $65^{\circ}$ angled emission spectra, these values increase to 0.74 and 0.86 , respectively.

Now, we make the technique significantly more surface sensitive using extreme-UV radiation and probe the shallow core level, Bi $5 d$; the experimental spectra are shown in Fig. 3. Each of the spin-orbit split peaks exhibit two distinct features, and their relative intensities strongly depend on the photon energy. In Fig. 3(b), we have superimposed the HAXPES data over the $49-\mathrm{eV}$ spectrum and find that the peak in the hard $\mathrm{x}$-ray spectrum matches the higher-binding-energy feature well; the linewidth of the HAXPES data is large due to larger resolution and lifetime broadenings of the final states. These observations suggest that the feature at higher binding energy is related to the bulk electronic structure, and the other one is the surface feature. The shift between surface and bulk features is found to be $0.27 \mathrm{eV}$. The presence of a two-peak structure in the UV spectra and a single peak in the HAXPES data has been observed in another material, $\mathrm{TlBiSe}_{2}$; the additional peak has been attributed to the surface clustering of the material, leading to a change in the effective valency of constituent atoms, which also gives rise to photoelectron diffraction [14]. The change in valency would shift the core levels of $\mathrm{Bi}$ and $\mathrm{Pd}$ in opposite direction. Here, we observe a different scenario: the energy shift is similar in both the Bi and Pd cases, indicating another origin for the difference in binding energies of the surface and bulk peaks; the energy 
shift of the Pd states is discussed later in relation to the valence band spectrum.

The 45-eV spectrum shows higher intensity of the bulk peak. With the increase in photon energy, the bulk peak becomes gradually weaker, and the surface peak gets stronger; the intensities of the surface and bulk peaks become almost similar at $49 \mathrm{eV}$, and the intensity ratio reverses above $49 \mathrm{eV}$. We have fit all the spectra using the Doniach-Šunjić line shape representing the features and find a good description in each case; representative fits are shown in Fig. 3(b). The line shapes of the Bi $5 d$ features are equally broad in bulk and surface. In generally, surface core level spectra can acquire additional width because the higher degree of disorder and/or reconstruction occurs at the surface [15]. Nearly equal widths $(\approx 0.3 \mathrm{eV})$ of the surface and bulk core level peaks suggest that the surface-bulk difference in disorder and/or any other surface effects such as reconstructions and impurities are negligible. The intensities of the constituent peaks represent the surface and bulk contributions to the photoemission signal and can be used to calculate the escape depth at different photon energies, as discussed below.

The photoemission intensity $I(\epsilon)$ can be expressed as $I(\epsilon)=\int_{0}^{d} I^{s}(\epsilon) e^{-d /[\lambda(\epsilon) \cos \theta]} d x+\int_{d}^{\infty} I^{b}(\epsilon) e^{-d /[\lambda(\epsilon) \cos \theta]} d x$. Here, $\lambda(\epsilon)$ is the mean escape depth at kinetic energy $\epsilon, d$ is the thickness of the surface layer, and $\theta$ is the emission angle with respect to the sample normal. $I^{s}(\epsilon)$ and $I^{b}(\epsilon)$ are the surface and bulk spectral functions, respectively. The first and second terms represent the contributions from surface and bulk electronic structures of the material. The intensity ratio of the surface and bulk contributions can be derived as $\frac{\text { Surface }}{\text { Bulk }}=e^{d / \lambda}-1$. Using this relation and the intensity of the component peaks, we estimate $\lambda / d$ and plot them in Fig. 3(c). $\lambda / d$ exhibits a gradual decrease with the increase in photon energy and thereby increase in the kinetic energy of photoelectrons. The minimum appears between $35-$ and $40-\mathrm{eV}$ kinetic energy, consistent with the findings in various other systems and the universal curve [16-18].

The valence band spectra collected using hard $\mathrm{x}$ rays and $35-\mathrm{eV}$ photon energy are shown in Fig. 4. The 35-eV spectra correspond to the electron kinetic energy close to the lowest escape depth and hence essentially provide the surface electronic structure, while the hard x-ray data represent the bulk electronic structure. For comparison, we show the calculated density of states using density functional theory. The 35-eV data exhibit two sharp peaks around 2.1- and 3.7-eV binding energies; these features match the calculated $\mathrm{Pd} 4 d$ partial density of states (PDOS) remarkably well. We do not see a significant contribution from the Bi $6 p$ PDOS in the $35-\mathrm{eV}$ spectrum due to the dominance of the photoemission cross section of $\mathrm{Pd} 4 d$ states $\sigma(\mathrm{Pd} 4 d)$ compared to the cross section of $\mathrm{Bi} 6 p$ states $\sigma(\mathrm{Bi} 6 p)$ (the atomic cross section ratio $\sigma(\mathrm{Bi} 6 p) / \sigma(\operatorname{Pd} 4 d) \approx 0.02$ [19]) in addition to the dominant PDOS of $\mathrm{Pd} 4 d$. The photoemission cross section of $\mathrm{Bi} 6 p$ becomes appreciable at hard $\mathrm{x}$-ray photon energy $(\sigma(\mathrm{Bi} 6 p) / \sigma(\mathrm{Pd} 4 d) \approx 0.1[20])$, and the experimental spectra exhibit significant intensity near the Fermi level and the 5-6-eV binding energy regime.

It is evident from the experimental spectra at two photon energies that the features in the hard X-ray data appear at

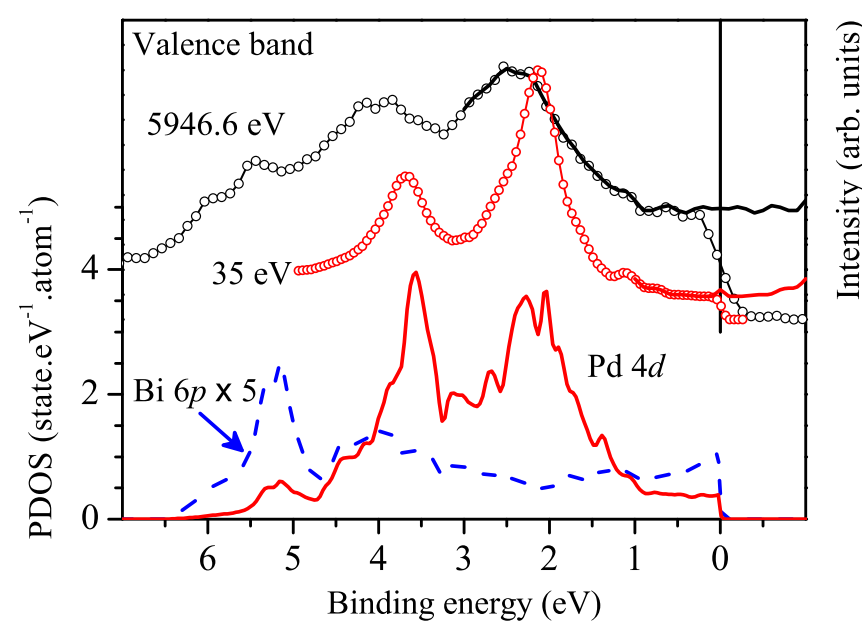

FIG. 4. Valence band spectra of BiPd collected with 5946.6 and $35 \mathrm{eV}$ at normal emission. The lines superimposed over the experimental data are the symmetrized data exhibiting flat intensity across the Fermi level. The lines in the lower panel are the calculated partial density of states of $\mathrm{Bi} 6 p$ (dashed line) and $\mathrm{Pd} 4 d$ (solid line) constituting the valence band. For clarity, Bi $6 p$ contributions to the formula unit are multiplied by 5 .

relatively higher binding energies compared to the $35-\mathrm{eV}$ data, as also found in the core level data. In order to investigate the spectral function in the vicinity of the Fermi level, we have symmetrized the data with respect to the Fermi level. The results are shown by lines superimposed over the corresponding experimental spectra. In both the cases, the spectral intensity is flat across the Fermi level, as expected from the calculated density of states.

From the above experimental results, one can make three conclusions: (i) the surface and bulk electronic structures of this system are different, (ii) the surface peaks in the core level spectra are shifted towards lower binding energy by $0.27 \mathrm{eV}$ relative to the bulk features, and (iii) the branching ratios of the Bi-related core level peaks are much larger than the ratio of the multiplicity of the peaks; the branching ratio of the Pd core level peaks matches their multiplicity ratio well.

The energy shift of the peaks can easily be explained by the surface effects as follows. Due to the translational symmetry breaking along the surface normal, the widths of the surface energy bands are expected to be narrower than those in the bulk electronic structure, as shown in a schematic diagram in Fig. 5. Since the Fermi level is pinned at the top of the $\mathrm{Pd} 4 d$ band (see the calculated results as a reference), a narrowing of the valence band would lead to a shift in the Fermi level to lower energies. The alignment of the Fermi levels of the bulk and surface bands leads to a shift in the core level peaks towards lower binding energies.

The angular momentum $J$ of the core hole created by photoexcitation is $J=L \pm S ; L$ and $S$ are the orbital and spin quantum numbers, respectively. The intensity of the peaks corresponding to each of the core hole states will depend on its multiplicity $(2 J+1)$, and hence, the branching ratio is $[2(L-S)+1]:[2(L+S)+1]$. This is observed to hold well in various core level spectroscopies. The deviation from such behavior suggests a paradigm shift from atomic descriptions 
(a)

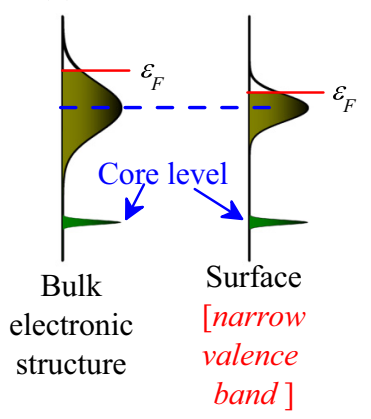

(c)

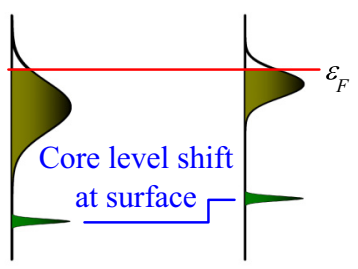

Fermi level of bulk and Surface electronic structures aligned (surface core level binding energy) becomes smaller than bulk)

FIG. 5. Schematic exhibiting the valence band and core level; the Fermi level is pinned at the top of the valence band. The surface valence band narrows down due to various surface effects. The alignment of the Fermi level emerges as a lowering of the binding energy of the core level peaks.

as the behavior of electrons in a solid is expected to be different. The orbital angular momentum gets significantly modified due to crystal momentum in a solid. In the relativistic description, the spin-orbit term for an electron in an external scalar potential $V(r)$ can be expressed as $-\frac{e \hbar}{(2 m c)^{2}} \sigma \cdot(E(r) \times$ $p)$. Here, $E(r)$ is the electric field $[=-\nabla V(r)]$, and $p$ is electron momentum. In a crystal without inversion symmetry, the potential gradient and hence the electric field will be finite, which will lead to appreciable angular momentum. This description helped to capture the electronic structure of noncentrosymmetric semiconductors such as GaAs, InSb, etc., and is known as the Dresselhaus effect [21]. Such an effect becomes significantly enhanced in heavier elements [22].

While core levels are believed to follow the atomic description, the translational symmetry in the solid will affect the dispersion of the core electronic states, although this is not documented in the literature to our knowledge. The absence of the center of symmetry in BiPd will lead to an enhancement of the orbital angular momentum, and hence, the effective $J$ in BiPd will be larger than the atomic value. This effect will be significantly stronger for Bi than for Pd (the atomic mass of $\mathrm{Bi}$ is close to double the atomic mass of $\mathrm{Pd}$ ). If

we take the branching ratio calculated for normal emission spectra, $L_{\text {eff }}$ for Bi $5 d$ is found to be about 2.4, and for $4 f$, it is 4.9; the larger increase for $4 f$ electrons indicates their higher sensitivity to the heavier mass of $\mathrm{Bi}$ as $4 f$ electrons possess more local character than the $5 d$ electrons. Such a scenario is consistent with the observation of enhancement of the branching ratio in the $65^{\circ}$ emission spectra, which is relatively more sensitive to the surface, and the potential gradient is expected to be enhanced at the surface, as found for the valence electrons in Rashba's description [23].

\section{CONCLUSION}

In summary, we studied the electronic structure of a noncentrosymmetric superconductor, BiPd, using high-resolution photoemission spectroscopy. Experiments were carried out on high-quality single crystals using hard x-ray and ultraviolet radiations at different experiment topologies. We showed that while HAXPES captured the bulk electronic structure well, the distinct differences between the surface and bulk electronic structure could be revealed using UV energies. The surface peaks appear at lower binding energy than the bulk peaks, which can be attributed to the narrowing of the surface electronic structure. We estimated the escape depth of photoelectrons as a function of electron kinetic energy, which exhibits the highest surface sensitivity near $40-\mathrm{eV}$ electron kinetic energy. Most interestingly, we discovered a deviation of the branching ratio of $\mathrm{Bi}$ core level peaks from the ratio of the final-state multiplicity calculated using the atomic description, while the Pd core levels exhibit atomic behavior. These results provide evidence of the breakdown of the atomic description of core level spectroscopy.

\section{ACKNOWLEDGMENTS}

The authors acknowledge financial support from the DSTDESY program and DAE, government of India, under Project No. 12-R\&D-TFR-5.10-0100. K.M. acknowledges financial assistance from the DST, government of India, under the J.C. Bose Fellowship program and the DAE, government of India, under the DAE-SRC-OI Award program.
[1] B. Joshi, A. Thamizhavel, and S. Ramakrishnan, Phys. Rev. B 84, 064518 (2011).

[2] Z. Sun, M. Enayat, A. Maldonado, C. Lithgow, E. Yelland, D. C. Peets, A. Yaresko, A. P. Schnyder, and P. Wahl, Nat. Commun. 6, 6633 (2015).

[3] L. Jiao, J. L. Zhang, Y. Chen, Z. F. Weng, Y. M. Shao, J. Y. Feng, X. Lu, B. Joshi, A. Thamizhavel, S. Ramakrishnan, and H. Q. Yuan, Phys. Rev. B 89, 060507(R) (2014).

[4] M. Mondal, B. Joshi, S. Kumar, A. Kamlapure, S. C. Ganguli, A. Thamizhavel, S. S. Mandal, S. Ramakrishnan, and P. Raychaudhuri, Phys. Rev. B 86, 094520 (2012).

[5] M. Neupane, N. Alidoust, M. Moffazel Hosen, J.-X. Zhu, K. Dimitri, S.-Y. Xu, N. Dhakal, R. Sankar, L. Belopolski, D. S. Sanchez, T.-R. Chang, H.-T. Jeng, K. Miyamoto, T. Okuda,
H. Lin, A. Bansil, D. Kaczorowski, F. Chou, M. Zahid Hasan, and T. Durakiewicz, Nat. Commun. 7, 13315 (2016).

[6] S. Thirupathaiah, S. Ghosh, R. Jha, E. D. L. Rienks, K. Dolui, V. V. Ravi Kishore, B. Buchner, T. Das, V. P. S. Awana, D. D. Sarma, and J. Fink, Phys. Rev. Lett. 117, 177001 (2016).

[7] H. M. Benia, E. Rampi, C. Trainer, C. M. Yim, A. Maldonado, D. C. Peets, A. Stohr, U. Starke, K. Kern, A. Yaresko, G. Levy, A. Damascelli, C. R. Ast, A. P. Schnyder, and P. Wahl, Phys. Rev. B 94, 121407(R) (2016).

[8] S. Doniach and M. Šunjić, J. Phys. C 3, 285 (1970).

[9] R. S. Singh and K. Maiti, Solid State Commun. 140, 188 (2006); Phys. Rev. B 76, 085102 (2007); K. Maiti and D. D. Sarma, ibid. 58, 9746 (1998).

[10] D. Biswas, S. Thakur, G. Balakrishnan and K. Maiti, Sci. Rep. 5, 17351 (2015). 
[11] R. Bindu, K. Maiti, S. Khalid, and E. V. Sampathkumaran, Phys. Rev. B 79, 094103 (2009); R. Bindu, K. Maiti, R. Rawat, and S. Khalid, Appl. Phys. Lett. 92, 121906 (2008); P. L. Paulose, N. Mohapatra, and E. V. Sampathkumaran, Phys. Rev. B 77, 172403 (2008); K. Maiti, R. S. Singh, V. R. R. Medicherla, S. Rayaprol, and E. V. Sampathkumaran, Phys. Rev. Lett. 95, 016404 (2005).

[12] S. de Jong, Y. Huang, R. Huisman, F. Massee, S. Thirupathaiah, M. Gorgoi, F. Schaefers, R. Follath, J. B. Goedkoop, and M. S. Golden, Phys. Rev. B 79, 115125 (2009); K. Maiti and D. D. Sarma, ibid. 54, 7816 (1996).

[13] J. C. Folmer and D. K. G. de Boer, Solid State Commun. 38, 1135 (1981).

[14] K. Kuroda, M. Ye, E. F. Schwier, M. Nurmamat, K. Shirai, M. Nakatake, S. Ueda, K. Miyamoto, T. Okuda, H. Namatame, M. Taniguchi, Y. Ueda, and A. Kimura, Phys. Rev. B 88, 245308 (2013).
[15] A. W. Newton, S. Haines, P. Weightman, and R. J. Cole, J. Electron Spectrosc. Relat. Phenom. 136, 235 (2004).

[16] M. P. Seah and W. A. Dench, Surf. Interface Anal. 1, 2 (1979).

[17] K. Maiti, U. Manju, S. Ray, P. Mahadevan, I. H. Inoue, C. Carbone, and D. D. Sarma, Phys. Rev. B 73, 052508 (2006); K. Maiti, P. Mahadevan, and D. D. Sarma, Phys. Rev. Lett. 80, 2885 (1998).

[18] S. Thakur, D. Biswas, N. Sahadev, P. K. Biswas, G. Balakrishnan and K. Maiti, J. Appl. Phys. 114, 053904 (2013).

[19] Numbers obtained from the information are available at https: //vuo.elettra.eu/services/elements/WebElements.html.

[20] J. J. Yeh and I. Lindau, At. Data Nucl. Data Tables 32, 1 (1985).

[21] G. Dresselhaus, Phys. Rev. 100, 580 (1955).

[22] J.-M. Jancu, R. Scholz, E. A. de Andrada e Silva, and G. C. La Rocca, Phys. Rev. B 72, 193201 (2005), and references therein.

[23] E. I. Rashba, Sov. Phys. Solid State 1, 368 (1959). 